\title{
Implementation of cell-free tumor DNA sequencing from the cerebrospinal fluid to guide treatment in a patient with primary leptomeningeal melanoma: A case report
}

\author{
JOHANNES C. MELMS ${ }^{1,2 *}$, KA-WAI HO ${ }^{3 *}$, ROHIT THUMMALAPALLI ${ }^{2,4}$, JANICE TYLER $^{5}$, \\ TITUS JOSEF BRINKER ${ }^{6}$, VEENA SINGH ${ }^{7}$, SOMA SENGUPTA ${ }^{3}$, JAMES MIER ${ }^{1}$ and BENJAMIN IZAR ${ }^{1,2,8}$ \\ ${ }^{1}$ Division of Hematology and Oncology, Beth Israel Deaconess Medical Center, Harvard Medical School; \\ ${ }^{2}$ Department of Medical Oncology, Dana-Farber Cancer Institute; ${ }^{3}$ Department of Neurology, Beth Israel \\ Deaconess Medical Center, Harvard Medical School; ${ }^{4}$ Harvard-MIT Division of Health Sciences and Technology, \\ Harvard Medical School; ${ }^{5}$ Department of Pathology, Beth Israel Deaconess Medical Center, Harvard Medical \\ School, Boston, MA 02215, USA; ${ }^{6}$ Department of Dermatology and National Center for Tumor Diseases, \\ University Hospital Heidelberg, University of Heidelberg, Heidelberg D-69120, Germany; ${ }^{7}$ BioCeptInc., \\ San Diego, CA 92121; ${ }^{8}$ Broad Institute of Harvard and MIT, Cambridge, MA 02139, USA
}

Received March 20, 2018; Accepted May 8, 2018

DOI: $10.3892 / \mathrm{mco} .2018 .1621$

\begin{abstract}
Primary leptomeningeal melanoma (PLM) is a rare type of cancer that represents a major clinical and molecular diagnostic challenge. A definitive diagnosis requires consistent magnetic resonance imaging findings and cerebrospinal fluid (CSF) cytology. Due to the small number of malignant cells in the CSF, routine testing for mutations in the $B R A F$ gene is difficult, which prevents the stratification of these patients to potentially beneficial therapies. We herein present the case of a 62-year old man with CSF cytology indicating PLM, where $B R A F$ mutation testing, from cell-free (cf) tumor DNA isolated from the CSF and plasma was implemented to guide clinical decision making. Testing for BRAFV600E mutation from the CSF and plasma was technically feasible, yielded concordant results, and guided the treatment for this patient. This case suggests that mutation testing of cfDNA isolated from the CSF is technically feasible and may guide therapy in cases where a tissue diagnosis is not possible for PLM and other malignancies with defined oncogenic driver mutations.
\end{abstract}

Correspondence to: Dr Benjamin Izar, Department of Medical Oncology, Dana-Farber Cancer Institute, 330 Brookline Avenue, Boston, MA 02215, USA

E-mail: benjamin_izar@dfci.harvard.edu

${ }^{*}$ Contributed equally

Key words: cell-free tumor DNA, DNA sequencing, cerebrospinal fluid, primary leptomeningeal melanoma, targeted therapy

\section{Introduction}

Melanoma is the deadliest form of skin cancer and continues to have an increasing incidence in the last decades (1). About 50\% of melanomas harbor a $\mathrm{T}$ to A substitution in codon 600 of the $B R A F$ gene, resulting in a substitution of Valine to Glutamic Acid (BRAFV600E), causing tonic activation of the RAF/MEK/ERK pathway, proliferation, and cell survival $(2,3)$. While a combination of selective RAF/MEK inhibitors results in brisk responses in most patients with BRAFV600E, treatment with these drugs in BRAF wild-type (WT) tumors leads to paradoxical activation of the pathway with the potential to accelerate tumor growth $(4,5)$. Determining the BRAF mutation status is therefore critical prior to initiation of RAF/MEK-inhibitors. In contrast, immune checkpoint inhibitors, which are monoclonal antibodies that target CTLA-4 (i.e., ipilimumab) or the PD-1/PD-L1 axis (e.g., nivolumab or pembrolizumab) exhibit activity irrespective of the BRAF mutation status, and are therefore the preferred first-line therapy for patients with BRAF WT melanoma (6-8). Testing for the BRAF mutation is usually performed on a tissue biopsy, such as a core needle biopsy. However, there are instances, in which potentially significant morbidity prohibits procedures for obtaining tissue. Primary leptomeningeal melanoma (PLM) is a rare and very aggressive type of melanoma with an estimated frequency of 1 in 10 million individuals (9-12). Due to its localization involving the letptomeninges, biopsies cannot be easily performed, which may limit potential therapeutic benefits for these patients. Here, we describe a case of a patient with PLM who underwent $B R A F$ mutation testing from cell-free DNA (cfDNA) isolated from cerebrospinal fluid (CSF) to guide therapy choices.

\section{Case report}

A 62-year-old Caucasian man with a past medical history of essential hypertension and obstructive sleep apnea was 
Table I. CSF cell count and chemistry from two LPs performed on hospital days 1 and 4.

\begin{tabular}{lrrrrrrrrr}
\hline LP no. & $\begin{array}{c}\text { WBC } \\
(/ \mu \mathrm{l})\end{array}$ & $\begin{array}{c}\text { RBC } \\
(/ \mu \mathrm{l})\end{array}$ & $\begin{array}{c}\text { PMN } \\
(\%)\end{array}$ & $\begin{array}{c}\text { LYM } \\
(\%)\end{array}$ & $\begin{array}{c}\text { MONO } \\
(\%)\end{array}$ & $\begin{array}{c}\text { MACRO } \\
(\%)\end{array}$ & $\begin{array}{c}\text { OTHER } \\
(\%)\end{array}$ & $\begin{array}{c}\text { Glucose } \\
(\mathrm{mg} / \mathrm{dl})\end{array}$ & $\begin{array}{c}\text { Protein } \\
(\mathrm{mg} / \mathrm{dl})\end{array}$ \\
\hline 1(HD1) & 28 & 16,750 & 42 & 48 & 7 & 1 & 4 & 60 & 516 \\
2(HD4) & 5 & 7,950 & 7 & 57 & 6 & 23 & 6 & 53 & 459 \\
Reference range & $<5$ & 0 & 0 & 0 & 0 & 0 & 0 & $45-80$ & $15-45$ \\
\hline
\end{tabular}

CSF, cerebrospinal fluid; LP, lumbar puncture; HD, hospital day; WBC, white blood cells; RBC, red blood cells; PMN, polymorphonuclear leukocytes; LYM, lymphocytes; MONO, monocytes; MACRO, macrophages.

admitted to Beth Israel Deaconess Medical Center with altered mental status, headaches and gait difficulties in March of 2016. Three months prior to presentation, the patient had noticed lower back pain with radiation to the buttocks. Magnetic resonance imaging (MRI) without contrast of the lumbar spine at that time was unrevealing. Over the following 6 weeks, the patient developed worsening gait difficulties and intermittent confusion and, 3 days prior to presentation, he developed headaches and was persistently confused.

On arrival to our emergency room, the patient was somnolent and only oriented to name. The vital signs were notable for a temperature of $102^{\circ} \mathrm{F}$, heart rate 74 beats $/ \mathrm{min}$, blood pressure $162 / 98 \mathrm{mmHg}$, respiratory rate 20 breaths/min, and oxygen saturation $97 \%$ at ambient air. Physical examination revealed somnolence with responses only to noxious stimuli, nuchal rigidity with a positive Brudzinski sign, and bilateral papilledema.An immediate non-contrast head computed tomography (CT) scan showed extensive communicating hydrocephalus and transependymal flow. The patient underwent a large-volume lumbar puncture where the opening pressure was $34 \mathrm{~cm} \mathrm{H}_{2} \mathrm{O}$, with subsequent mental status improvement. A complete cell count and chemistry of the CSF is summarized in Table I. The patient was admitted to the neurological intensive care unit for further care.

An MRI scan of the head and spine with and without contrast revealed diffuse leptomeningeal enhancement involving intracranial and spinal meninges on post-contrast T1-weighted imaging, as well as communicating hydrocephalus. There was no parenchymal central nervous system (CNS) involvement (Fig. 1A-C). A CT scan of the chest, abdomen and pelvis did not reveal evidence of visceral metastatic disease. A full skin examination by a dermatologist reported no evidence of a primary cutaneous melanoma, and the ophthalmic examination did not reveal any suspicious lesions. Cytology of the CSF revealed malignant cells with strong staining for human melanoma black-45 (HMB-45), confirming a diagnosis of malignant melanoma (Fig. 1D and E).

Overall, this presentation was consistent with a primary leptomeningeal melanoma (PLM). The patient was treated with dexamethasone and required emergent placement of an external ventricular drain (EVD) due to interval worsening mental status in the setting of hydrocephalus. CSF was collected for isolation of cfDNA and sequencing of the $B R A F$ gene. Plasma was collected at the same time for cfDNA sequencing. BRAF mutation testing of CSF and plasma was performed using the Biocept Target Selector ${ }^{\mathrm{TM}}$ assay.
The patient received five fractions of whole-brain radiation therapy (2,000 cGy) and palliative radiation of the spine from level T12 to S3. He had significant improvement of his neurological symptoms, allowing for removal of the EVD on day 12 of his hospitalization, and was discharged from the hospital on day 20.

Although previous efforts have used targeted next-generation sequencing to evaluate small panels of genes involved in melanoma biology, including $B R A F$ and $N R A S$ (13), only mutant $B R A F$ is a currently actionable target and may help guide the choice of targeted therapy vs. immunotherapy. Sequencing of cfDNA revealed wild-type (WT) BRAF gene in both the CSF and plasma. Based on this finding, treatment with either ipilimumab, a PD-1 checkpoint inhibitor, or temozolomide was discussed. Given the patient's good clinical status, treatment with ipilimumab was initiated, with a plan to administer four cycles, potentially followed by PD-1 checkpoint blockade. Although the patient received his first infusion without treatment-related complications, the course was complicated by the development of a pulmonary embolism, which delayed a planned second infusion. Five weeks after the first ipilimumab infusion $(\sim 9$ weeks after the initial diagnosis), the patient developed rapidly progressing confusion and gait instability with worsening hydrocephalus and succumbed to the disease 3 days later.

\section{Discussion}

Primary leptomeningeal melanoma (PLM) is a very rare type of cancer that is considered to arise from melanocytes in the pia and arachnoid (10). Diagnostic criteria for PLM have been suggested (9), including hyperintensity of the meninges on T1-weighted MRI and cytology with positive immunostaining for lineage-specific HMB-45 and S-100 (14-16).

In $\sim 25 \%$ of patients, PLM is associated with giant melanocytic nevi, which frequently carry treatment-sensitizing oncogenic driver mutations $(17,18)$ including $B R A F V 600 E$ and NRASQ61. Among patients with metastatic cutaneous melanoma, $\sim 50 \%$ harbor sensitizing $B R A F$ mutations, most commonly BRAFV600E. Treatment with RAF or $\mathrm{RAF} / \mathrm{MEK}$-inhibitors in this subset of patients has resulted in unprecedented response rates and improvement of progression-free and overall survival $(4,19)$. However, patients with wild-type $B R A F$ melanoma are not candidates for RAF/MEK inhibition, as BRAF inhibitors may promote growth of $B R A F-\mathrm{WT}$ cells and further exacerbate 


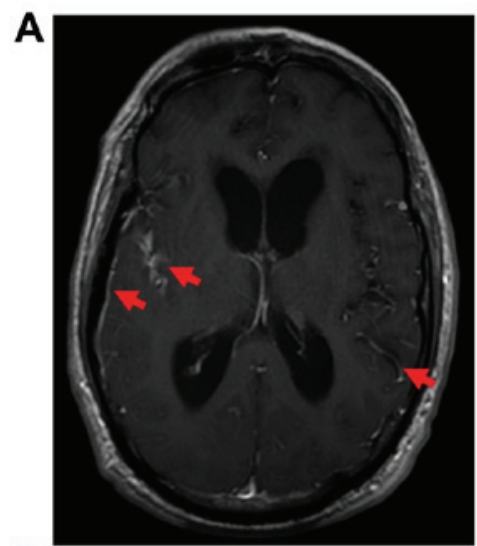

B
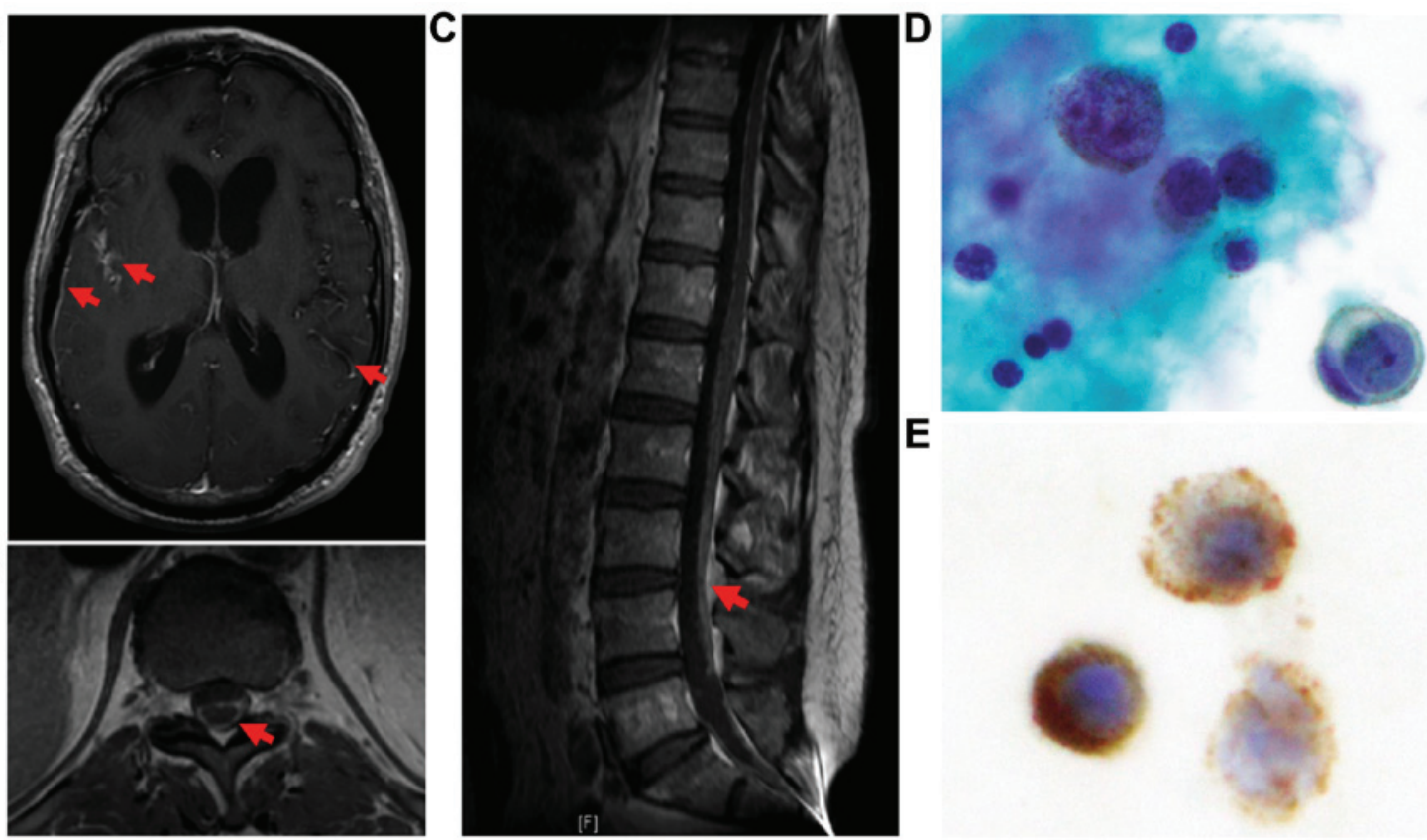

Figure 1. Imaging and CSF pathology revealed PLM with CSF involvement. (A) MRI of the brain with and without contrast: Post-contrast axial T1 sequence. Diffuse leptomeningeal enhancement (arrows) with enlarged third ventricle. (B) MRI of the thoracic spine post-contrast axial T1; arrow indicates leptomeningeal enhancement. (C) MRI of the thoracolumbar spine post-contrast sagittal T1; arrow indicates leptomeningeal enhancement. Cytological analysis of the CSF including (D) ThinPrep and (E) HMB-45 staining. Examination of the CSF revealed dyshesive large atypical cells with high nuclear-to-cytoplasmic ratio, macronucleoli, and fine cytoplasmic pigment. Cannibalistic cells and occasional mitotic figures were present. Immunohistochemical stains performed on cytospin preparations were strongly positive for HMB-45 and negative for cytokeratin, confirming involvement of the CSF by malignant melanoma. CSF, cerebrospinal fluid; PLM, primary leptomeningeal melanoma; MRI, magnetic resonance imaging; HMB-45, human melanoma black-45.

the disease (20), highlighting the importance of targeted $B R A F$ testing in this patient. In patients with $B R A F-\mathrm{WT}$ melanoma, first-line immunotherapies are currently under investigation as an alternative strategy. To this end, the Food and Drug Administration has approved immunotherapies, such as the CTLA-4 inhibitor ipilimumab and PD-1 checkpoint inhibitors, including nivolumab and pembrolizumab, as first-line therapies for patients with metastatic melanoma. Single-agent treatment with any of these compounds or combinations of ipilimumab and PD-1 inhibitors produce deep and long-lasting responses in a subset of patients $(6-8,21)$, including those with leptomeningeal disease (22).

The ideal choice of first-line therapy, RAF/MEK-inhibitors or immunotherapies, in patients with $B R A F$-mutant melanoma remains unclear and is mostly guided by the clinical setting. For example, in patients with rapidly progressing $B R A F$-mutant melanoma, treatment with BRAF/MEK inhibitors may induce faster responses and is the preferred treatment modality. In patients without sensitizing $B R A F$ mutations ( $B R A F-\mathrm{WT})$, as in the present case, immunotherapy is the first-line treatment.

Understanding the molecular profile of these tumors is crucial for employing treatments such as targeted therapies or immune checkpoint inhibitors, which may induce dramatic responses in leptomeningeal melanoma $(22,23)$. However, testing from malignant CSF with as few as 1 malignant cell per $\mu \mathrm{l}$, as in the case presented here, is challenging with current approaches. We herein report the successful use of targeted $B R A F$ sequencing of cfDNA isolated from the CSF as well as the plasma in a patient with PLM. The results from $B R A F$ mutation testing were instrumental in selecting the treatment for this patient, given the potential harm in treating a $B R A F$-WT patient with RAF/MEK inhibitors. Recent reports indicating the feasibility of molecular profiling from CSF (24-27) have mostly focused on primary CNS tumors. With regard to cfDNA sequencing from CSF for melanoma, previous reports have focused only on monitoring treatment response in metastatic lesions for which the genomic status of the primary melanoma lesion was known $(28,29)$. In contrast, our case displays the utility of using cfDNA to guide treatment choice in a primary leptomeningeal melanoma for which no genetic information was available. This study indicates that rapid targeted sequencing of cfDNA from the CSF is clinically feasible and should be considered for guiding treatment in patients in whom a tissue biopsy cannot be obtained, including those with PLM and leptomeningeal metastatic disease.

\section{Acknowledgements}

We thank Lyle Arnold, PhD and Cecile Rose Vibat, PhD from Biocept Inc. for providing technical support.

\section{Funding}

BI is supported by the National Cancer Institute (K08CA222663) and the Ludwig Center for Cancer Research at Harvard.

\section{Availability of data and materials}

All data generated or analyzed during this study are included in this published article. 


\section{Authors' contributions}

JCM, KH, TJB, SS, JM and BI took clinical care of the patient. JT provided pathology slides. JCM, RT and BI wrote the paper. All authors read, reviewed and approved the manuscript.

\section{Ethics approval and consent to participate}

Not applicable.

\section{Consent for publication}

The family agreed to publication of the case and material presented here.

\section{Competing interests}

VS is an employee of BioCept Inc. The other authors declare that they have no competing interests.

\section{References}

1. Siegel RL, Miller KD and Jemal A: Cancer statistics, 2017. CA Cancer J Clin 67: 7-30, 2017.

2. Hodis E, Watson IR, Kryukov GV, Arold ST, Imielinski M, Theurillat JP, Nickerson E, Auclair D, Li L, Place C, et al: A landscape of driver mutations in melanoma. Cell 150: 251-263, 2012.

3. Cancer Genome Atlas Network: Genomic classification of cutaneous melanoma. Cell 161: 1681-1696, 2015.

4. Flaherty KT, Puzanov I, Kim KB, Ribas A, McArthur GA, Sosman JA, O'Dwyer PJ, Lee RJ, Grippo JF, Nolop K and Chapman PB: Inhibition of mutated, activated BRAF in metastatic melanoma. N Engl J Med 363: 809-819, 2010.

5. Poulikakos PI, Zhang C, Bollag G, Shokat KM and Rosen N: RAF inhibitors transactivate RAF dimers and ERK signalling in cells with wild-type BRAF. Nature 464: 427-430, 2010.

6. Hodi FS, O'Day SJ, McDermott DF, Weber RW, Sosman JA, Haanen JB, Gonzalez R, Robert C, Schadendorf D Hassel JC, et al: Improved survival with ipilimumab in patients with metastatic melanoma. N Engl J Med 363: 711-723, 2010.

7. Larkin J, Chiarion-Sileni V, Gonzalez R, Grob JJ, Cowey CL, Lao CD, Schadendorf D, Dummer R, Smylie M, Rutkowski $\mathrm{P}$, et al: Combined nivolumab and ipilimumab or monotherapy in untreated melanoma. N Engl J Med 373: 23-34, 2015.

8. Robert C, Schachter J, Long GV, Arance A, Grob JJ, Mortier L, Daud A, Carlino MS, McNeil C, Lotem M, et al: Pembrolizumab versus ipilimumab in advanced melanoma. N Engl J Med 372: 2521-2532, 2015

9. Hayward RD: Malignant melanoma and the central nervous system. A guide for classification based on the clinical findings. J Neurol Neurosurg Psychiatry 39: 526-530, 1976.

10. Rosenthal G, Gomori JM, Tobias S, Diment J and Shoshan Y: Unusual cases involving the CNS and nasal sinuses: Case 1. Primary leptomeningeal melanoma. J Clin Oncol 21: 3875-3877, 2003.

11. Paulus W and Hasselblatt M: Tumoren. In: Neuropathologie. Springer, Berlin-Heidelberg, pp481-549, 2012.

12. Hsieh YY, Yang ST, Li WH, Hu CJ and Wang LS: Primary leptomeningeal melanoma mimicking meningitis: A case report and literature review. J Clin Oncol 33: e57-e61, 2015.

13. van de Nes J, Gessi M, Sucker A, Möller I, Stiller M, Horn S, Scholz SL, Pischler C, Stadtler N, Schilling B, et al: Targeted next generation sequencing reveals unique mutation profile of primary melanocytic tumors of the central nervous system. J Neurooncol 127: 435-444, 2016.
14. Wick MR, Swanson PE and Rocamora A: Recognition of malignant melanoma by monoclonal antibody HMB-45. An immunohistochemical study of 200 paraffin-embedded cutaneous tumors. J Cutan Pathol 15: 201-207, 1988.

15. Tosaka M, Tamura M, Oriuchi N, Horikoshi M, Joshita T, Sugawara K, Kobayashi S, Kohga H, Yoshida T and Sasaki T: Cerebrospinal fluid immunocytochemical analysis and neuroimaging in the diagnosis of primary leptomeningeal melanoma. Case report. J Neurosurg 94: 528-532, 2001.

16. Sagiuchi T, Ishii K, Utsuki S, Asano Y, Tsukahara S, Kan S, Fujii K and Hayakawa K: Increased uptake of technetium-99m-hexamethylpropyleneamine oxime related to primary leptomeningeal melanoma. AJNR Am J Neuroradiol 23: 1404-1406, 2002.

17. Hoffman HJ and Freeman A: Primary malignant leptomeningeal melanoma in association with giant hairy nevi. J Neurosurg 26: 62-71, 1967

18. Salgado CM, Basu D, Nikiforova M, Bauer BS, Johnson D, Rundell V, Grunwaldt LJ and Reyes-Múgica M: BRAF mutations are also associated with neurocutaneous melanocytosis and large/giant congenital melanocytic nevi. Pediatr Dev Pathol 18: $1-9,2015$

19. Flaherty KT, Robert C, Hersey P, Nathan P, Garbe C, Milhem M, Demidov LV, Hassel JC, Rutkowski P, Mohr P, et al: Improved survival with MEK inhibition in BRAF-mutated melanoma. N Engl J Med 367: 107-114, 2012.

20. Medina TM and Lewis KD: The evolution of combined molecular targeted therapies to advance the therapeutic efficacy in melanoma: A highlight of vemurafenib and cobimetinib. Onco Targets Ther 9: 3739-3752, 2016.

21. Hamid O, Robert C, Daud A, Hodi FS, Hwu WJ, Kefford R, Wolchok JD, Hersey P, Joseph RW, Weber JS, et al: Safety and tumor responses with lambrolizumab (anti-PD-1) in melanoma. N Engl J Med 369: 134-144, 2013.

22. Margolin K, Ernstoff MS, Hamid O, Lawrence D, McDermott D, Puzanov I, Wolchok JD, Clark JI, Sznol M, Logan TF, et al: Ipilimumab in patients with melanoma and brain metastases: An open-label, phase 2 trial. Lancet Oncol 13: 459-465, 2012.

23. Wilgenhof S and Neyns B: complete cytologic remission of V600E BRAF-mutant melanoma-associated leptomeningeal carcinomatosis upon treatment with dabrafenib. J Clin Oncol 33: e109-e111, 2015.

24. Pan W, Gu W, Nagpal S, Gephart MH and Quake SR: Brain tumor mutations detected in cerebral spinal fluid. Clin Chem 61: 514-522, 2015.

25. Wang Y, Springer S, Zhang M, McMahon KW, Kinde I, Dobbyn L, Ptak J, Brem H, Chaichana K, Gallia GL, et al: Detection of tumor-derived DNA in cerebrospinal fluid of patients with primary tumors of the brain and spinal cord. Proc Natl Acad Sci USA 112: 9704-9709, 2015

26. De Mattos-Arruda L, Mayor R, Ng CK, Weigelt B, Martínez-Ricarte F, Torrejon D, Oliveira M, Arias A, Raventos C, Tang J, et al: Cerebrospinal fluid-derived circulating tumour DNA better represents the genomic alterations of brain tumours than plasma. Nat Commun 6: 8839, 2015.

27. Pentsova EI, Shah RH, Tang J, Boire A, You D, Briggs S, Omuro A, Lin X, Fleisher M, Grommes C, et al: Evaluating cancer of the central nervous system through next-generation sequencing of cerebrospinal fluid. J Clin Oncol 34: 2404-2415, 2016.

28. Li Y, Pan W, Connolly ID, Reddy S, Nagpal S, Quake S and Gephart MH: Tumor DNA in cerebral spinal fluid reflects clinical course in a patient with melanoma leptomeningeal brain metastases. J Neurooncol 128: 93-100, 2016.

29. Momtaz P, Pentsova E, Abdel-Wahab O, Diamond E, Hyman D, Merghoub T, You D, Gasmi B, Viale A and Chapman PB: Quantification of tumor-derived cell free DNA(cfDNA) by digital PCR (DigPCR) in cerebrospinal fluid of patients with BRAFV600 mutated malignancies. Oncotarget 7: 85430-85436, 2016. 